approaches into research plans. When a consultation is requested, MICHR's Community Engagement (CE) Program responds by planning a meeting with staff and faculty who have relevant skills, expertise, and connections. After the initial meeting, the CE Program provides follow-up communication and support based on the needs of the specific request, and often facilitates connections with potential partners. RESULTS/ANTICIPATED RESULTS: The two most frequent types of consultation requests involve 1) making connections with potential researchers or community partner organizations, and 2) providing guidance on research grant applications that involve community engagement. MICHR provides approximately 50 CEnR consultations each year, which have resulted in development of new partnerships, grant submissions, and research projects that utilize CEnR principles and address community-identified health priorities. DISCUSSION/SIGNIFICANCE OF IMPACT: This presentation will describe the evolution of MICHR's CEnR consultation process and highlight successful outcomes and lessons learned over its 12-year history. CONFLICT OF INTEREST DESCRIPTION: NA

4046

\section{Museum and Arts-Space Programming Intended to Improve Health: Interim Survey Results}

Ian Koebner ${ }^{1}$, Helen Chatterjee ${ }^{2}$, Daniel J. Tancredi ${ }^{1}$, Claudia M. Witt $^{3}$, Pier Luigi Sacco ${ }^{4}$, Ruchi Rawal ${ }^{1}$, and Fred J Meyers ${ }^{1}$ ${ }^{1}$ University of California, Davis; ${ }^{2}$ University College London; ${ }^{3}$ University of Zurich; ${ }^{4}$ IULM University

OBJECTIVES/GOALS: Many museums and art spaces conduct programming intended to improve health outcomes, but arts professionals' perceptions of these programs are not well known. This study describes arts professionals' experiences with museum and art-space interventions intended to improve health. METHODS/STUDY POPULATION: A 14-item digital Qualtrics survey was administered to museums and arts organizations selected using snowball sampling. The survey was sent to a range of arts and cultural organizations and professional membership bodies in the US and UK. Survey questions assessed the range of audiences involved in health programs, what types of activities museums and arts organizations are offering to support health outcomes, and how programs are evaluated. RESULTS/ ANTICIPATED RESULTS: From 10/30/19-11/19/19, 151 surveys were completed; 66 respondents (44\%) have a museum/arts in health program. Common target populations include individuals with mental health concerns $(33,22 \%)$ and older adults $(26,17 \%)$. Improving wellbeing $(56,37 \%)$ and social isolation $(50,33 \%)$ were the most common intended outcomes. Respondents reported using a variety of program evaluation methods including formal $(23,15 \%)$, informal $(31,21 \%)$, and anecdotal $(37,25 \%)$. Interviews are planned with a purposive sample of respondents conducting, or interested in conducting, a program for individuals with chronic pain and those formally evaluating their programs. DISCUSSION/SIGNIFICANCE OF IMPACT: Interim survey responses indicate many cultural organizations engage in programming intended to improve health outcomes. Understanding the cultural sector's current efforts to improve health represents an initial step in translating these efforts into effective intersectoral research partnerships.
4073

Parents of Faith Support School-Based Sex Education: A Louisiana Study

Elise Walsh Boos ${ }^{1}$, Caitlin Canfield, $\mathrm{MPH}^{2}$, Lissane Brown, PhD, $\mathrm{MPH}^{3}$, and Kristie Bardell, $\mathrm{MPH}^{2}$

${ }^{1}$ Albert Einstein College of Medicine; ${ }^{2}$ Louisiana Public Health Institute; ${ }^{3} \mathrm{Abt}$ Associates

OBJECTIVES/GOALS: Louisiana state law does not require sex-education (SE) in public schools. Locally and nationally, religious identity and beliefs are often invoked to oppose access to sexual and reproductive healthcare and education. This study aimed to explore support for SE among Louisiana parents, focusing on how religiosity may influence parent support for SE. METHODS/STUDY POPULATION: Participants included 1,197 Louisiana parents and caregivers of children in grades K-12 who completed a web-based survey. Multivariate logistic regression analysis was used to determine associations between covariates and support for SE. RESULTS/ANTICIPATED RESULTS: Sixty-eight percent of parents reported that their overall approach to life is based on their religion or faith. Of those parents, $77 \%$ agreed that schools should be required to offer SE. In multivariate analysis, parents who reported that their whole approach to life is based on their religion on faith were $26 \%$ less likely to support required SE compared to parents whose whole approach to life was not based on their religion; however, this difference was not statistically significant (adjusted OR 0.74, 95\% CI 0.44 1.24). Although support for required SE declined as religiosity increased, a strong majority of parents support requiring SE in Louisiana schools, regardless of religiosity. DISCUSSION/ SIGNIFICANCE OF IMPACT: Contrary to opposition claims, strong support for SE exists among Louisiana parents and caregivers of faith. Parents and leaders of faith may be engaged as partners in advocacy for SE as well as other sexual and reproductive health issues.

4301

Racial/Ethnic variations in discharge destination after inpatient care: A risk-adjusted analysis of a large regional dataset $^{\dagger}$

Arnab Kumar Ghosh ${ }^{1}$, and Said Ibrahim ${ }^{1}$

${ }^{1}$ Weill Cornell Medical College of Cornell University

OBJECTIVES/GOALS: While there are many well-documented factors for racial/ethnic variation in discharge destination, less is known about the role hospital processes play. We hypothesize that variation in hospital processes -defined as the patient length of stay (LOS) adjusted for known confounders - explains racial/ethnic variation in discharge destination. METHODS/STUDY POPULATION: Our sample was 176,686 discharges from 165 hospitals in 2014 using the New York State Inpatient Dataset from the Healthcare Cost and Utilization Project, merged with the 2014 American Hospital Association Annual Survey to build a file of inpatient discharges with patient, disease and socio-economic characteristics. We excluded patients under 18 years, those with LOS of zero, those who died, those admitted to critical access hospitals, and patients from hospitals that lacked sufficient number of minority patients. We used a generalized linear mixed effects model to create an in-hospital risk-adjusted LOS by modelling the relationship between the interaction of race and discharge destination and LOS, controlling for known confounders such as patient, disease and between-hospital 